\title{
Inspection of maleic anhydride in starch powder using line-scan hyperspectral Raman chemical imaging technique
}

\author{
Jianwei Qin ${ }^{1}$, Moon S. Kim ${ }^{1 *}$, Kuanglin Chao ${ }^{1}$, Lisa Bellato ${ }^{2}$, Walter F. Schmidt ${ }^{1}$, \\ Byoung-Kwan $\mathrm{Cho}^{3}$, Min Huang ${ }^{4}$ \\ (1. USDA/ARS Environmental Microbial and Food Safety Laboratory, Beltsville Agricultural Research Center, 10300 Baltimore Ave., \\ Beltsville, MD 20705, USA; 2. Department of Production Engineering, Municipal Education Foundation of Piracicaba (FUMEP) - \\ Piracicaba's School of Engineering, 560 Monsenhor Martinho Salgot Ave., Piracicaba, SP 13414-040, Brazil; \\ 3. Department of Biosystems Machinery Engineering, College of Agricultural and Life Science, Chungnam National University, \\ 99 Daehak-ro, Yuseoung-gu, Daejeon 305-764, South Korea; 4. Key Laboratory of Advanced Process Control for Light Industry \\ (Ministry of Education), Jiangnan University, 1800 Lihu Avenue, Wuxi 214122, Jiangsu, China)
}

\begin{abstract}
Excessive use of maleic anhydride (MAN) in starch production is potentially harmful for consumers' health. This study presents a macro-scale Raman chemical imaging method for detection and quantification of MAN particles mixed in starch powder. MAN was mixed into corn starch at eight concentration levels from $50 \mathrm{ppm}$ to $6400 \mathrm{ppm}$ (w/w). Each mixture was put in a sample holder with a $150 \mathrm{~mm} \times 100 \mathrm{~mm}$ area and a $2 \mathrm{~mm}$ depth to create a large surface and a thin layer of the powdery sample for inspection. A $30 \mathrm{~W} 785 \mathrm{~nm}$ line laser was projected on the sample surface, from which hyperspectral images were obtained by a line-scan Raman imaging system with a spatial resolution of $0.2 \mathrm{~mm}$. Fluorescence signals generated by laser-sample interactions were eliminated by a mathematical baseline correction method. A unique Raman peak was selected at $1839 \mathrm{~cm}^{-1}$ for the MAN detection, at which single-band fluorescence-corrected images were extracted from the mixture of each concentration and used to generate chemical images for MAN detection and mapping. The MAN detection limit was estimated at $100 \mathrm{ppm}$ based on the Raman imaging measurement results. Pixel concentrations of the MAN in the chemical images were found linearly correlated with mass concentrations of the MAN particles in the starch powder, suggesting the Raman chemical imaging method has the potential for quantitative detection of the MAN in the starch-MAN mixtures.
\end{abstract}

Keywords: Raman spectroscopy, chemical imaging, starch, adulteration, food authentication

DOI: $10.25165 /$ j.ijabe.20181106.4339

Citation: Qin J W, Kim M S, Chao K L, Bellato L, Schmidt W F, Cho B-K, et al. Inspection of maleic anhydride in starch powder using line-scan hyperspectral Raman chemical imaging technique. Int J Agric \& Biol Eng, 2018; 11(6): 120-125.

\section{Introduction}

Natural starch exists in a great number of plant roots, tubers, and seeds for the purpose of energy storage. To improve its physical properties (e.g., viscosity, texture, and stability) for food consumption purposes, modified starch is manufactured by chemical processing of the natural starch that is extracted and refined from the plant crops during starch production. Since the starch is contained in a large amount of staple foods (e.g., corn, wheat, rice, and potato) and is the most common carbohydrate consumed by humans, the safety and quality of the starch is a critical issue in the world. Illegal chemical additives, which are

Received date: $2018-04-20 \quad$ Accepted date: 2018-07-25

Biographies: Jianwei Qin, PhD, Agricultural Engineer, research interests: sensing technologies for food safety, Email: jianwei.qin@ars.usda.gov; Kuanglin Chao, $\mathrm{PhD}$, Agricultural Engineer, research interests: sensing technologies for food safety, Email: kevin.chao@ars.usda.gov; Lisa Bellato, undergraduate student, research interests: food production engineering, Email: lisa_bellato@hotmail.com; Walter F. Schmidt, PhD, Research Chemist, research interests: structural elucidation and spectroscopy of phase transitions, Email: walter.schmidt@ars.usda.gov; Byoung-Kwan Cho, PhD, Professor, research interests: nondestructive biosensing, Email: chobk@cnu.ac.kr; Min Huang, PhD, Professor, research interests: sensing technologies for food quality, Email: huangmzqb@163.com.

*Corresponding author: Moon S. Kim, PhD, Research Physicist, research interests: sensing technologies for food safety. USDA/ARS/EMFSL, Bldg. 303, BARC-East, 10300 Baltimore Ave., Beltsville, MD 20705-2350, USA. Tel: +1-301-504-8462; Fax: +1-301-504-9466; Email: moon.kim@ars.usda.gov. intentionally added to the starch during the manufacturing process, are a potential contaminant source that threatens the health of the general public. In 2013, abusive use of maleic anhydride (MAN) was found in production of the modified starch in Taiwan to improve chewiness, glutinosity, and water retention of the related food products ${ }^{[1]}$. The MAN is an industrial material that is produced mainly for coating and polymer applications, and it is an indirect food additive approved by the U.S. FDA that can be used as packaging materials in contact with food. However, the MAN is not intended to be added directly to food, and it has not been approved for use in the manufactured starch as a legal food additive in Taiwan. Once consumed by humans and contacted with water in an acidic environment of the stomach, the MAN is turned into maleic acid (MA) in a rapid hydrolysis reaction, which can pass through kidney cells to induce a kidney disease known as Fanconi syndrome $^{[2]}$. The MAN modified starch (i.e., starch maleate) had been involved in producing many types of foods (e.g., rice noodle, meat ball, and tofu pudding) in Taiwan, China. As a consequence, the "toxic starch" incident caused a fear to the public health and became a local and international image crisis for the Taiwanese food industry as numerous tainted food products had been recalled and destroyed during the incident.

Few studies have been reported to develop efficient and effective detection methods to inspect maleic anhydride and maleic acid mixed in starch powder. High-performance liquid chromatography-tandem mass spectrometry (HPLC-MS) techniques have been used to determine the amount of the MAN 
and the MA in the $\operatorname{starch}^{[3,4]}$. Liquid solutions need to be prepared to dissolve the starch powder samples for the measurements using the chromatography based methods. The time-consuming sample preparation procedures and the relatively small sampling volumes prevent the HPLC-MS methods from being used for high-throughput screening for the starch powder, despite their high detection accuracies and low detection limits. Similar detection method was also used to analyze trace level of the MA in urine and serum samples of MA-treated rats with an aim to investigating the potential adverse health effects for those consuming the foods containing the contaminated $\operatorname{starch}^{[2]}$. Optical sensing techniques (e.g., spectroscopy and imaging) have been investigated for safety and quality inspection of the food powders ${ }^{[5]}$, which are a promising alternative to the chromatography based techniques for direct authentication of the starch powder. Examples of the optical methods for composition analysis of the starch include near-infrared (NIR) spectroscopy for detection of adulterants in kudzu starch ${ }^{[6]}$ and quantitative analysis of maleic acid powder in cassava $\operatorname{starch}^{[7]}$, and Raman spectroscopy for determination of amylose content in corn and cassava starches ${ }^{[8]}$ and structure analysis and crystallinity calculation of corn starch ${ }^{[9]}$.

Most chemical contaminants, adulterants, and additives are Raman active, and their unique features characterized by narrow and sharp spectral peaks in Raman spectra can be used to detect the chemicals mixed in powdered foods and ingredients. Our group has developed macro-scale Raman chemical imaging techniques for direct inspection of various food powders and ingredients. Two point-scan hyperspectral Raman imaging systems were developed using $785 \mathrm{~nm}^{[10]}$ and $1064 \mathrm{~nm}^{[11]}$ point lasers, respectively. The point-scan systems have been used to detect chemical adulterants mixed in the food powders, such as melamine in milk powder ${ }^{[12]}$ and metanil yellow in turmeric powder ${ }^{[11]}$. A more efficient line-scan hyperspectral Raman imaging system using a $785 \mathrm{~nm}$ line laser was also developed to implement high-throughput chemical imaging for food safety research ${ }^{[13,14]}$. The line-scan system has been used for screening and quantitative analysis of the chemical contaminants mixed in the food powders, such as detection of melamine and urea mixed in milk powder ${ }^{[15]}$ and benzoyl peroxide mixed in wheat flour ${ }^{[16]}$. This study intended to follow our previous investigation on starch powder authentication $^{[17]}$, with the objective of utilizing the line-scan macro-scale Raman imaging method to directly inspect and quantify the MAN particles mixed in the starch powder at low concentration levels.

\section{Materials and methods}

\subsection{Hyperspectral Raman imaging system}

Figure 1 shows a photograph of the line-scan high-throughput hyperspectral Raman imaging system used in this study ${ }^{[13]}$. A $5 \mathrm{~W} 785 \mathrm{~nm}$ line laser used in our previous studies ${ }^{[15,16]}$ was upgraded to a $30 \mathrm{~W} 785 \mathrm{~nm}$ line laser (OptiGrate, Oviedo, FL, USA) to enhance the Raman scattering signals. In this new laser, light generated by two spatially combined diode laser heads passes through a $785 \mathrm{~nm}$ laser clean-up filter and two cylindrical lenses to form a divergent laser line. The laser line generation method by the fixed cylindrical lenses is different from the scanning mirror method (moving part) used by the previous $5 \mathrm{~W}$ line laser. To have the line laser normally projected on the sample, a $45^{\circ} 785 \mathrm{~nm}$ dichroic beamsplitter was used to change the direction of the laser propagation from horizontal to vertical. On the sample surface, the laser line is around $200 \mathrm{~mm}$ long and $2 \mathrm{~mm}$ wide. The Raman detection unit mainly includes a C-mount lens with $23 \mathrm{~mm}$ focal length, a Raman imaging spectrograph (ImSpector R10E, Specim, Oulu, Finland), and a CCD camera (iKon-M 934, Andor Technology, South Windsor, CT, USA). After passing through the beamsplitter, the lens, and the imaging spectrograph, the Raman scattering signals from the laser line are eventually acquired by the CCD camera. Two $785 \mathrm{~nm}$ long-pass filters are used to block the useless Rayleigh and anti-Stokes scattering signals generated from laser-sample interactions. Meanwhile, the CCD $(1024 \times 1024$ pixels) is thermoelectrically cooled to $-65^{\circ} \mathrm{C}$ to diminish the dark current during the Raman image acquisition. Using the $23 \mathrm{~mm}$ lens, the instantaneous field of view of the imaging system was determined as $205 \mathrm{~mm}$ long with a spatial resolution of $0.2 \mathrm{~mm} /$ pixel. A computer-controlled positioning stage is used to move the sample incrementally across the laser line, by which the system conducts hyperspectral Raman image acquisition using a line-scan method. The system covers a Raman shift wavenumber region up to $2831 \mathrm{~cm}^{-1}$ with a spectral resolution of $14 \mathrm{~cm}^{-1}$. In-house developed LabVIEW software is used for system control and image acquisition.

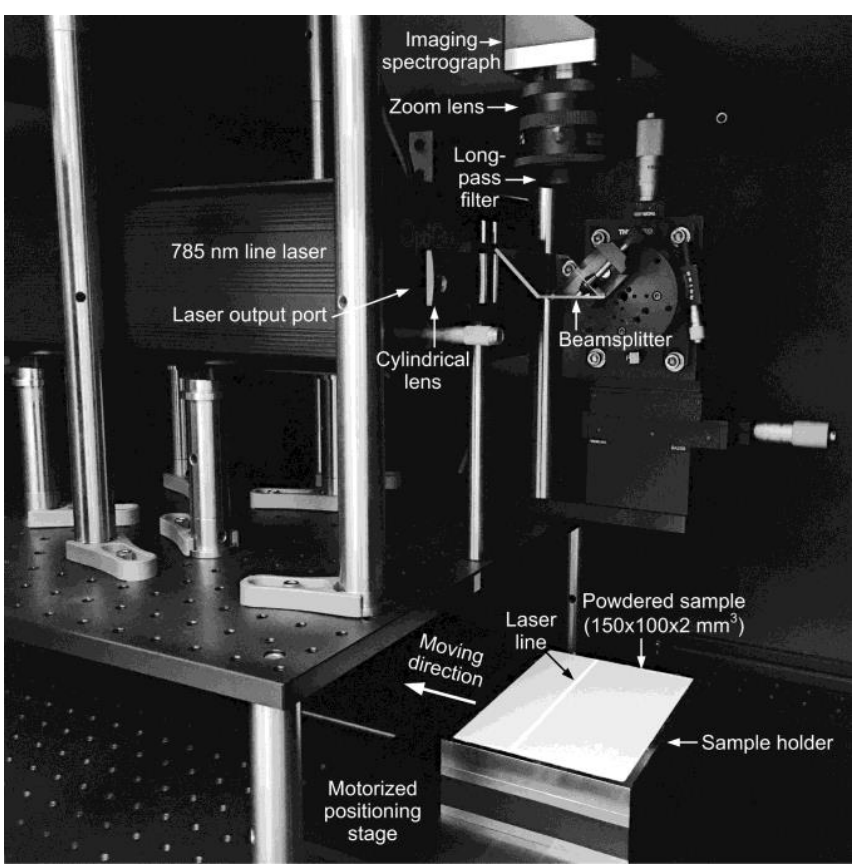

Figure 1 Hyperspectral image acquisition from starch powder samples using a line-scan macro-scale Raman imaging system

\subsection{Sample preparation and experimental procedures}

Maleic anhydride (>95\%) (Sigma-Aldrich, St. Louis, MO, USA) was grinded into fine particles using a mortar and a pestle before mixing. The fine MAN particles were then mixed into corn starch powder (ACH Food Companies, Memphis, TN, USA) at eight concentration levels (w/w): 6400, 3200, 1600, 800, 400, 200,100 , and $50 \mathrm{ppm}$ (or $\mathrm{mg} / \mathrm{kg}$ ). To make the MAN particles evenly distributed in the starch powder, a bench-top powder mixer (LabRAM, Resodyn Acoustic Mixers, Butte, MT, USA), which generates high-intensity acoustic waves to the samples utilizing a mechanical resonator, was used to mix the eight starch-MAN samples. The mass of each starch-MAN mixture was $22 \mathrm{~g}$, which was mixed for $5 \mathrm{~min}$ in a $16 \mathrm{oz}$. polystyrene vessel that was fixed by a vessel fixture of the mixer. A nickel plated container was used to hold the powdered sample at each concentration to minimize Raman and fluorescence signals from the sample holder. The sample volume is $150 \times 100 \times 2 \mathrm{~mm}^{3}$ in the container (Figure 1), which created a large sample area and a thin sample layer to ensure 
the MAN particles can be detected everywhere in the starch powder. During the hyperspectral Raman image collection, each sample was line scanned 500 times with a spatial incremental size of $0.2 \mathrm{~mm}$. As a result, a Raman hypercube with a dimension of $750 \times 500 \times 846$ was obtained for every mixture in a wavenumber range of $103-2831 \mathrm{~cm}^{-1}$ (846 bands). Image data below $103 \mathrm{~cm}^{-1}$ (i.e., $785-791 \mathrm{~nm}$ ) was not used due to laser interference signals and also lack of useful Raman information for chemical detection in this narrow region. The original sample images were subtracted by a dark current image acquired with the lens covered by an end cap. Further data analysis was only conducted to the subtracted images. In addition to the mixed samples, Raman spectra of pure corn starch and MAN were also collected as references.

\subsection{Raman data processing}

Figure 2 summarizes the Raman data analysis algorithms for quantitative detection of maleic anhydride particles mixed in corn starch powder. A unique Raman peak of MAN was first determined by comparing the reference spectra of pure corn starch and MAN. For the starch-MAN mixtures, the original Raman spectrum extracted from each hyperspectral pixel was smoothed by a first-order Savitzky-Golay filter to remove the high-frequency noise. A baseline correction method based on adaptive iteratively reweighted penalized least squares (airPLS) ${ }^{[18]}$ was then used to correct the fluorescence signals for all the smoothed Raman spectra of each sample. At the wavenumber of the selected MAN peak, single-band images were obtained from the fluorescence-corrected hyperspectral Raman images. A simple thresholding method using a single threshold was applied to the single-band fluorescence-free images to classify all the pixels to MAN adulterant particles or corn starch background. To minimize false positive and negative errors in the image pixel classifications, the Raman peaks of the MAN at the classified pixels were verified to determine the optimal threshold value for the final binary images. In addition, pixel concentrations of each sample were obtained by calculating the percentages of the MAN pixels in the chemical images (each image with a spatial dimension of $750 \times 500$ pixels). Linear regression analysis was then applied to the pixel and mass concentrations of the MAN to explore the possibility for quantifying the amount of the MAN particles in the starch powder using their chemical images. In-house developed MATLAB programs were used to implement the Raman data processing methods described above.

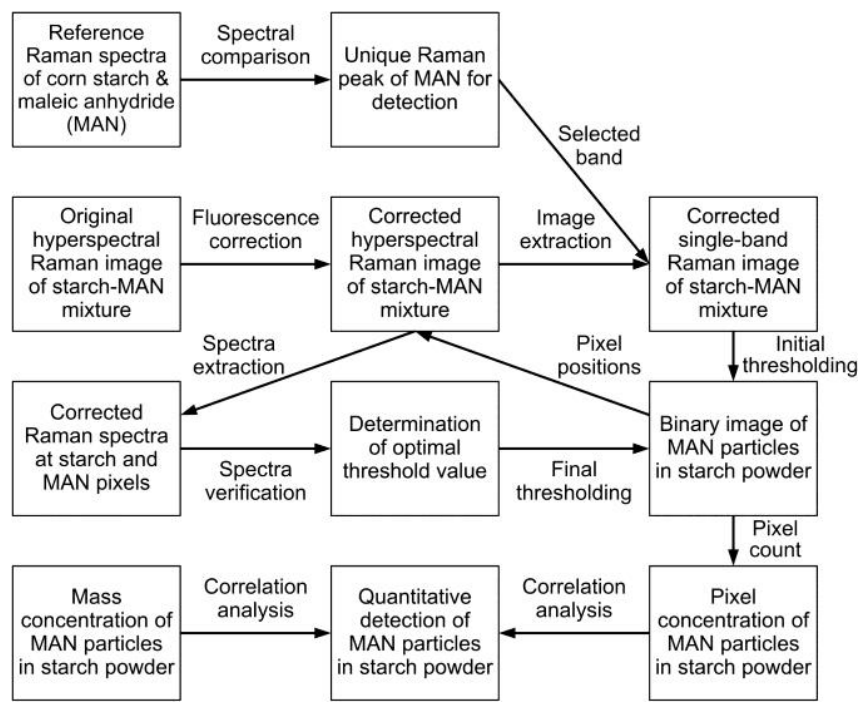

Figure 2 Data analysis procedures for inspection of maleic anhydride in starch powder

\section{Results and discussion}

\subsection{Raman spectra of corn starch and maleic anhydride}

Reference Raman spectra collected from pure corn starch and maleic anhydride in the wavenumber range of $103-2831 \mathrm{~cm}^{-1}$ are shown in Figure 3. Both corn starch and MAN exhibit fluorescence backgrounds in their original Raman spectra. The corn starch shows a couple of Raman peaks on top of the fluorescence baseline in the short wavenumber region of 103$1500 \mathrm{~cm}^{-1}$, which can be attributed to the constituents (e.g., amylose, amylopectin, and carbohydrates) in the $\operatorname{starch}^{[8]}$. Other brands and types of the starches, such as arrowroot starch, potato starch, and tapioca starch, show similar Raman spectral features with the corn starch tested in this study (data not shown). Meanwhile, several discrete Raman peaks were observed in the spectrum of the MAN, in which the strongest peak was shown at the wavenumber of $1839 \mathrm{~cm}^{-1}$, which is attributed to $\mathrm{C}=\mathrm{O}$ stretching in the $\mathrm{MAN}^{[19]}$. The structure of the corn starch contains no $\mathrm{C}=\mathrm{O}$ carbonyl groups and correspondingly no vibrational modes of $\mathrm{C}=\mathrm{O}$ stretching from ketone or carboxylic acids. As a result, no notable Raman peaks were observed near $1839 \mathrm{~cm}^{-1}$ for the corn starch as well as other brands and types of the starches. Since the $1839 \mathrm{~cm}^{-1}$ peak of the MAN does not overlap with any Raman peaks of the starch, it was determined as a unique peak wavenumber for the detection of the MAN in the starch.

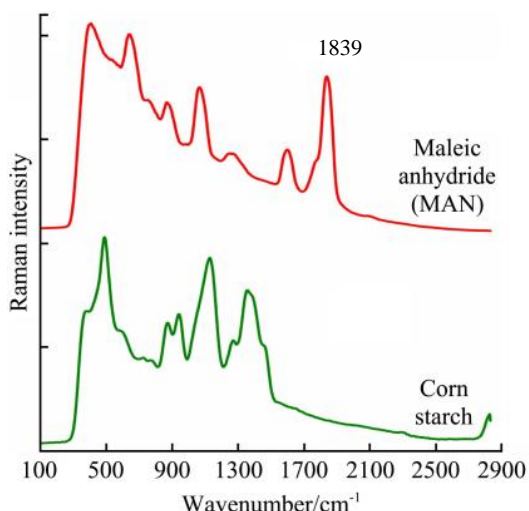

Figure 3 Raman spectra of corn starch and maleic anhydride

\subsection{Raman spectral processing and image pixel classification}

Figure 4 shows Raman image and spectral processing results for a $1600 \mathrm{ppm}$ starch-MAN sample. Original and fluorescencecorrected single-band Raman images at $1839 \mathrm{~cm}^{-1}$ are shown in Figure 4a and Figure 4b, respectively. Three pairs of pixels were selected and marked in the original and corrected images, at which three pairs of original (Figure 4d) and fluorescence-corrected Raman spectra (Figure 4e) were plotted to illustrate the representative spectral pattern of the clean starch and the starch contaminated by the MAN. The $1839 \mathrm{~cm}^{-1}$ peak of the MAN was shown in the original Raman spectrum at pixel "Mo", revealing the presence of the MAN particles in the corn starch. The fluorescence intensities of the corn starch at $1839 \mathrm{~cm}^{-1}$ (e.g., spectrum at pixel "Lo") are generally lower than those of the Raman peaks from the MAN. However, some starch particles (e.g., spectrum at pixel "Ho") exhibited strong fluorescence intensities that are higher than those of the starch-MAN mixtures (e.g., spectrum at pixel "Mo"). Therefore, the fluorescence correction is necessary to avoid false positive errors (i.e., misclassify starch as MAN) if the original Raman data are used for the MAN detection. The fluctuating fluorescence background 
signals were removed in the fluorescence-corrected Raman spectra, as demonstrated by three spectra in Figure 4e, which were vertically shifted for clarity and easy comparison. At $1839 \mathrm{~cm}^{-1}$, the Raman peak intensity of the corn starch with the MAN (at pixel
"Mc") is clearly higher than those of the pure starch (at pixels "Hc" and "Lc"), which formed a base for detecting the MAN by applying a simple thresholding method to the fluorescence-free images at $1839 \mathrm{~cm}^{-1}$.
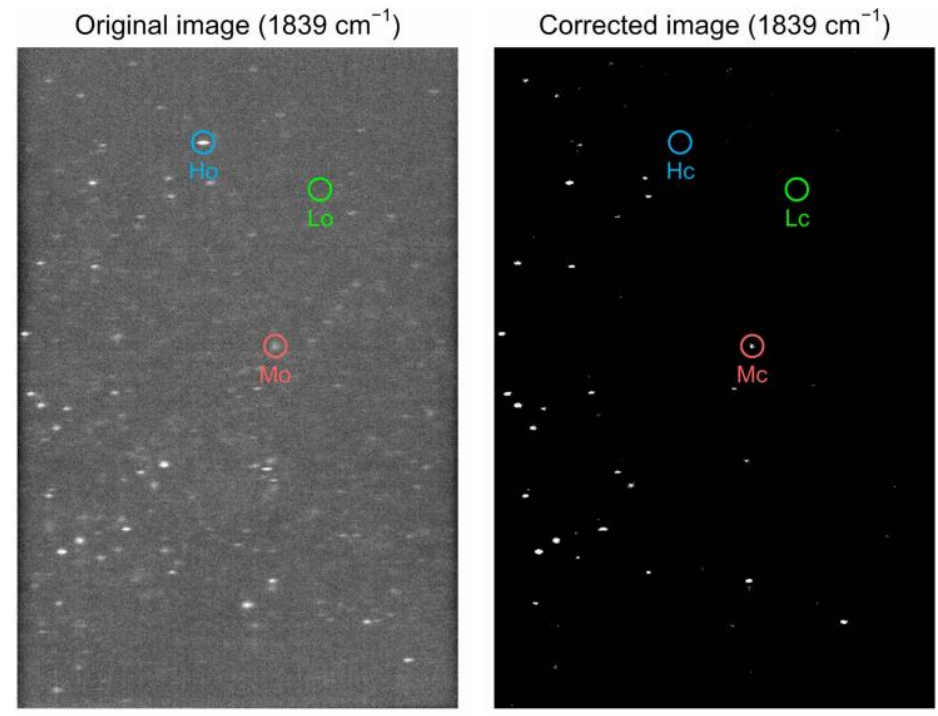

a. original image at $1839 \mathrm{~cm}^{-1}$

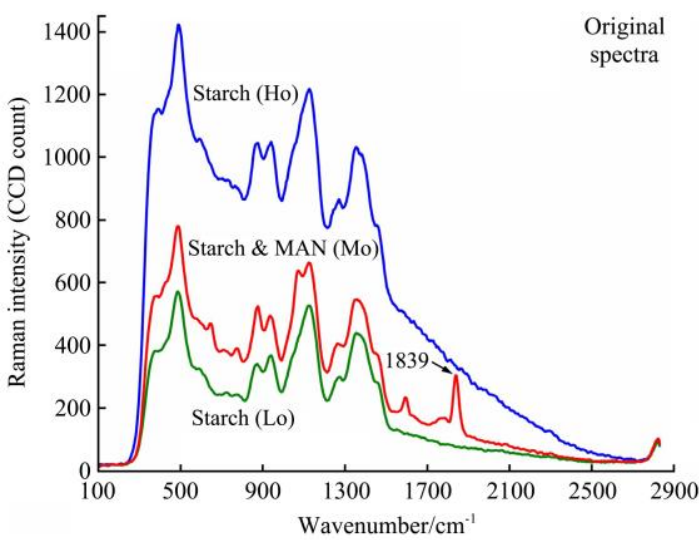

d. original spectra at selected pixels marked in Figure 4a

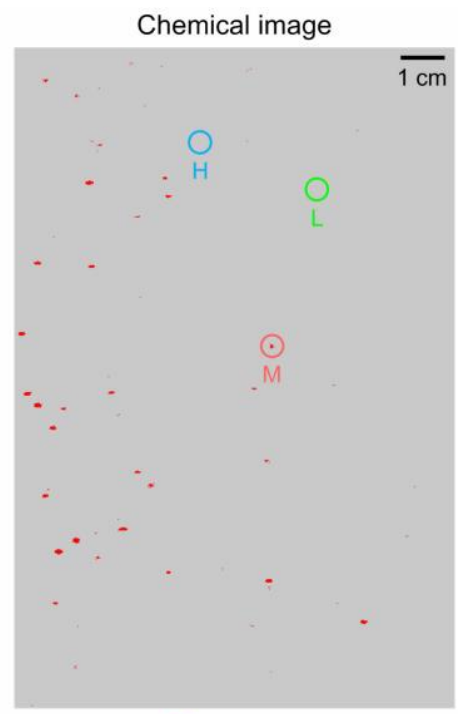

$\square$ Corn starch $\square$ Maleic anhydride (MAN) c. chemical image of the MAN

Figure 4 Raman image and spectral processing for a 1600 ppm starch-MAN mixture

The airPLS baseline correction method was used to correct the original Raman image in Figure $4 \mathrm{a}$, in which the fluorescence signals were removed at every hyperspectral pixel. The corrected image at $1839 \mathrm{~cm}^{-1}$ (Figure 4b) exhibited a consistent dark background after fluorescence removal. Since the Raman peak of the MAN at $1839 \mathrm{~cm}^{-1}$ is in the low-fluorescence spectral region of the starch, the MAN particles can be observed in the original Raman image at $1839 \mathrm{~cm}^{-1}$ (Figure 4a). However, the MAN pixels were mixed with some high-fluorescence starch pixels (e.g., pixel "Ho"). In the corrected image (Figure 4b), the bright fluorescence pixels of the corn starch were effectively removed (e.g., pixel "Hc"). Meanwhile, the relative intensities of the MAN pixels were clearly enhanced (e.g., pixel "Mc"), which made the MAN particles the only notable feature in the image. For the Raman images of the starch-MAN mixtures at other concentrations, similar results were found to eliminate the fluorescence-bright starch pixels and boost the Raman-bright MAN pixels. A binary image (Figure 4c) for the MAN detection was then generated by applying a simple thresholding method to the $1839 \mathrm{~cm}^{-1}$ corrected image (Figure 4b). The pixels with intensities higher than the threshold were identified as the MAN particles, while the remaining pixels were identified as the starch particles. Note that the $1839 \mathrm{~cm}^{-1}$ Raman peak uniquely selected for the MAN detection was used to determine the type of the adulterant in the chemical image. The MAN particles mixed in the corn starch can be clearly visualized in the final chemical image.

\subsection{Detection and quantitative analysis of maleic anhydride in corn starch}

Hyperspectral Raman images of all the starch-MAN mixtures were processed using the fluorescence correction and image pixel classification procedures demonstrated above. The final chemical images of the MAN in the starch powder are shown in Figure 5 to illustrate the particle features and the spatial distribution of the MAN at eight concentrations. As shown in the images, the MAN particles with different sizes were detected in the mixtures, which in general were broadly spread in the corn starch powder. The MAN pixel number gradually decreased with the decreasing mass concentration of the MAN. The MAN particles were detected in all the mixtures except for the lowest concentration of the $50 \mathrm{ppm}$. There are a total of 33 pixels that were classified as the MAN in the $100 \mathrm{ppm}$ sample. The $1839 \mathrm{~cm}^{-1}$ Raman peak of the MAN was observed in all 33 
corresponding spectra (data not shown, similar to the spectrum at pixel "Mc" in Figure 4e), suggesting no false positive errors occurred for the MAN pixel classifications. The $50 \mathrm{ppm}$ sample
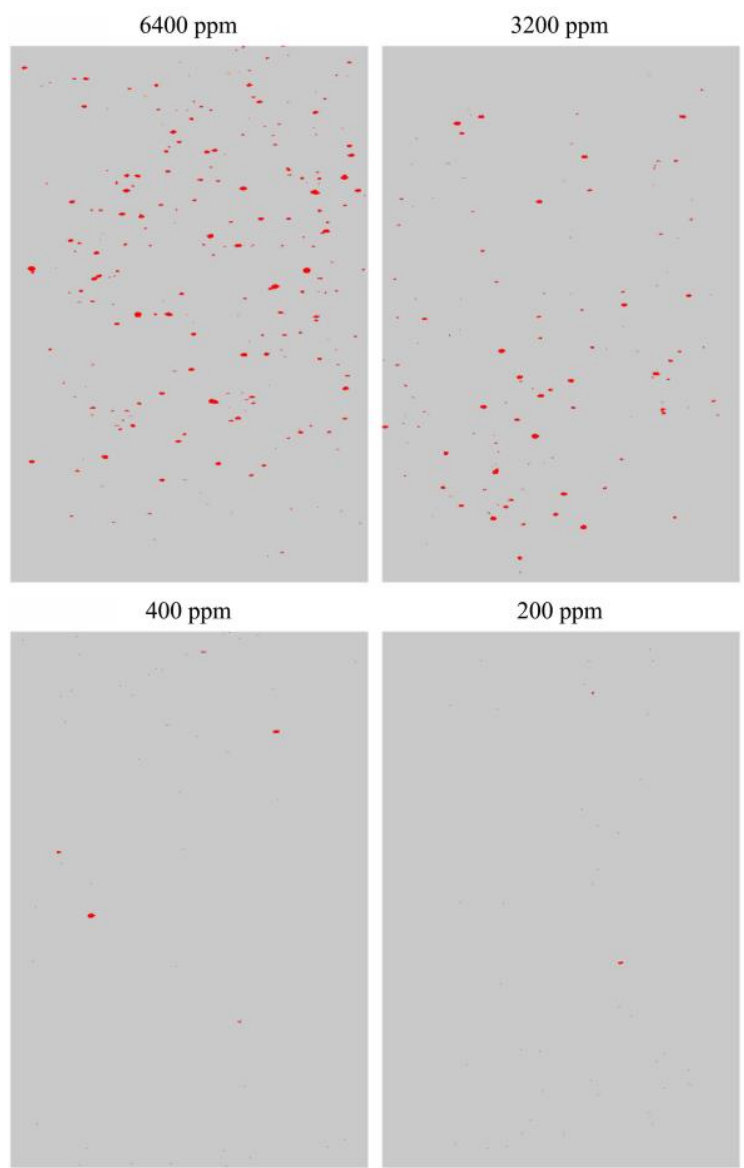

$200 \mathrm{ppm}$

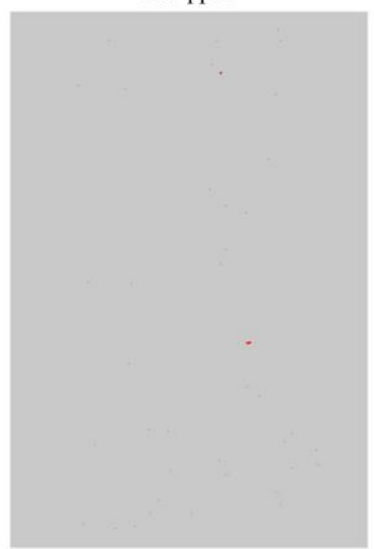

Figure 5 Chemical images of maleic anhydride in starch powder at different concentrations

In our previous studies, $50 \mathrm{ppm}$ LOD was obtained for melamine and urea in milk powder ${ }^{[15]}$ and benzoyl peroxide in wheat flour ${ }^{[16]}$. Possible reasons for the higher LOD for the MAN in the starch include relatively low Raman signal intensities of the MAN compared to other chemical adulterants (e.g., melamine, urea, and benzoyl peroxide) and different MAN-starch particle interactions during mixing (e.g., different particle pores and inter-particle voids with other food and adulterant powders). The LOD for the MAN using Raman imaging method in this study is lower than that of the NIR spectroscopy method (e.g., $6000 \mathrm{ppm}$ LOD for maleic acid powder in starch reported in [7]) and higher than that of the HPLC-MS method (e.g., 10 ppm LOD reported in [4]). The Raman chemical imaging method can be used for rapid high-throughput screening of the starch powder, which is not realistic for the HPLC-MS method due to time-consuming sample preparations and small sampling volumes.

For the starch-MAN mixtures at seven concentrations from 100 to $6400 \mathrm{ppm}$, the pixel concentrations of the MAN in the chemical images were obtained, which were then correlated to the mass concentrations for the quantitative analysis of the MAN particles in the corn starch. For instance, the $100 \mathrm{ppm}$ and $6400 \mathrm{ppm}$ mixed samples had 33 and 2872 MAN pixels in their chemical images, respectively. The MAN pixel concentrations for the two mixtures were determined as $33 /(750 \times 500)=88 \mathrm{ppm}$ and $2872 /(750 \times 500)=7659 \mathrm{ppm}$. Figure 6 plots the result of correlation analysis between the pixel concentrations and the mass concentrations of the MAN. It was found that the pixel did not have any pixels classified as the MAN in its chemical image. Hence the limit of detection (LOD) for the MAN mixed in the corn starch was estimated at $100 \mathrm{ppm}$.
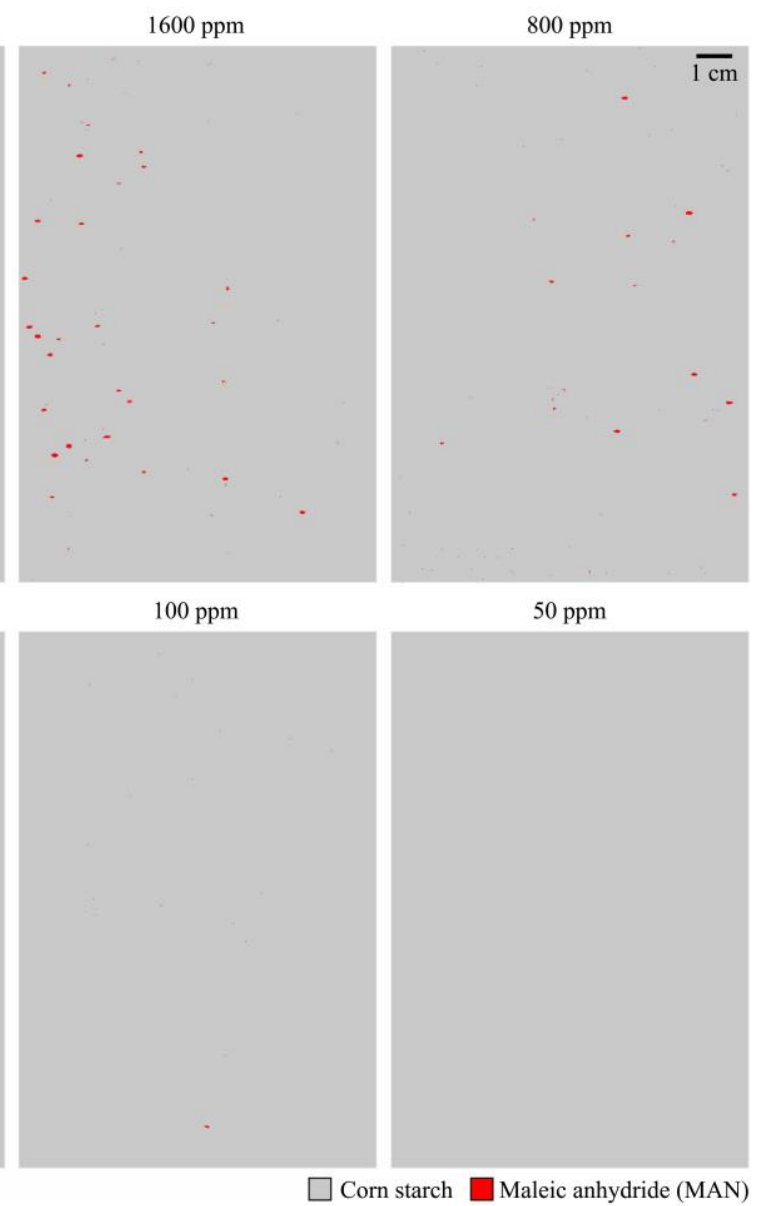

concentrations were linearly correlated with the mass concentrations of the MAN, with a correlation coefficient of 0.9994 The linear regression model can be used for quantitative detection of the MAN particles in the corn starch. The method for detecting the MAN in the starch powder used in this study, along with our previous studies on detecting melamine and urea in milk powder ${ }^{[15]}$

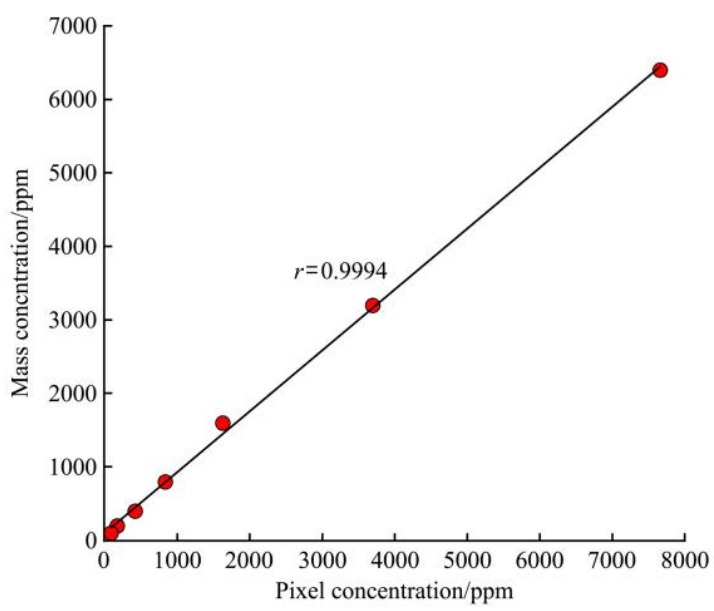

Figure 6 Linear regression analysis for pixel and mass concentrations of maleic anhydride in starch powder and benzoyl peroxide in wheat flour ${ }^{[16]}$, demonstrated that the line-scan Raman chemical imaging technique is promising to inspect different types of Raman-active contaminants, adulterants or illegal additives mixed in different powdered foods and 
ingredients. As the next step of this research, tests will be conducted on more adulterated food powders and replicates to verify measurement repeatability and build stable and reliable classification and quantification models.

\section{Conclusions}

This study presented a line-scan macro-scale Raman chemical imaging method for direct detection and quantification of maleic anhydride particles mixed in the powdery corn starch. The corn starch shows varying fluorescence signals when excited by the $785 \mathrm{~nm}$ line laser. The fluorescence baseline correction procedure can help avoid false positive errors for the MAN detection. The single-band image approach based on a preselected unique Raman peak of the MAN provides a targeted method to detect a particular adulterant in the starch. The particles of the MAN can be detected in the corn starch with a detection limit of $100 \mathrm{ppm}$, demonstrating the potential of the Raman imaging method for inspection of the starch powder in practice. The concentrations of the MAN pixels identified in the chemical images are linearly correlated with the mass concentrations of the MAN particles mixed in the starch powder, which can be used to evaluate the quantity of the MAN in the starch-MAN mixtures. The measurement concept based on macro-scale Raman images presented in this research can be generalized to detect various chemical contaminants, adulterants, and illegal additives mixed in a broad range of the food powders.

\section{[References]}

[1] Peng J, Chang M, Fang M, Liao C, Tsai C, Tseng S, et al. Incidents of major food adulteration in Taiwan between 2011 and 2015. Food Control, 2017; 72: 145-152.

[2] Chen $\mathrm{H}, \mathrm{Wu} \mathrm{C}, \mathrm{Wu} \mathrm{K}$. Determination of the maleic acid in rat urine and serum samples by isotope dilution-liquid chromatography-tandem mass spectrometry with on-line solid phase extraction. Talanta, 2015; 136: 9-14.

[3] Xu D, Chen Y, Zhou S, Lian Y, Chen L, Lin L, et al. Determination of the total amount of maleic acid and maleic anhydride in starch and its products by high performance liquid chromatography-tandem mass spectrometry. Chin. J. Chromatogr, 2013; 31(12): 1224-1227.

[4] Tsai C, Wu G, Kuo C, Lin Y, Chang C, Tseng S, et al. Effective extraction method through alkaline hydrolysis for the detection of starch maleate in foods. J. Food Drug Anal., 2015; 23(3): 442-446.

[5] Su W, Sun D. Fourier transform infrared and Raman and hyperspectral imaging techniques for quality determinations of powdery foods: a review. Compr. Rev. Food Sci. Food Saf., 2018; 17(1): 104-122.

[6] Xu L, Shi W, Cai C, Zhong W, Tu K. Rapid and nondestructive detection of multiple adulterants in kudzu starch by near infrared (NIR) spectroscopy and chemometrics. LWT Food Sci. Technol., 2015; 61(2): 590-595.

[7] Fu H, Li H, Xu L, Yin Q, Yan T, Ni C, et al. Detection of unexpected frauds: Screening and quantification of maleic acid in cassava starch by Fourier transform near-infrared spectroscopy. Food Chem, 2017; 227: 322-328.

[8] Almeida M R, Alves R S, Nascimbem L B L R, Stephani R, Poppi R J, de Oliveira L F C. Determination of amylose content in starch using Raman spectroscopy and multivariate calibration analysis. Anal. Bioanal. Chem., 2010; 397: 2693-2701.

[9] Liu Y, Xu Y, Yan Y, Hu D, Yang L, Shen R. Application of Raman spectroscopy in structure analysis and crystallinity calculation of corn starch. Starch - Stärke, 2015; 67(7-8): 612-619.

[10] Qin J, Chao K, Kim M S. Raman chemical imaging system for food safety and quality inspection. Trans. ASABE, 2010; 53(6): 1873-1882.

[11] Chao K, Dhakal S, Qin J, Kim M S, Peng Y. A 1064 nm dispersive Raman spectral imaging system for food safety and quality evaluation. Appl. Sci., 2018; 8(3): 431.

[12] Dhakal S, Chao K, Qin J, Kim M S, Chan D. Raman spectral imaging for quantitative contaminant evaluation in skim milk powder. J. Food. Meas. Charact., 2016; 10: 374-386.

[13] Qin J, Chao K, Cho B, Peng Y, Kim M S. High-throughput Raman chemical imaging for rapid evaluation of food safety and quality. Trans. ASABE 2014; 57: 1783-1792.

[14] Qin J, Chao K, Kim M S, Cho B. Line-scan macro-scale Raman chemical imaging for authentication of powdered foods and ingredients. Food Bioprocess Technol., 2016; 9: 113-123.

[15] Qin J, Kim M S, Chao K, Dhakal S, Lee H, Cho B, et al. Detection and quantification of adulterants in milk powder using a high-throughput Raman chemical imaging technique. Food Addit. Contam. Part A, 2017; 34(2): 152-161.

[16] Qin J, Kim M S, Chao K, Gonzalez M, Cho B. Quantitative detection of benzoyl peroxide in wheat flour by line-scan macro-scale Raman chemical imaging. Appl. Spectrosc., 2017; 71(11): 2469-2476.

[17] Qin J, Kim M S, Chao K, Bellato L. Detecting maleic anhydride in starch using line-scan hyperspectral Raman chemical imaging. ASABE Annual International Meeting, Spokane, WA. USA. 2017; ASABE Paper, No. 1700398.

[18] Zhang Z, Chen S, Liang Y. Baseline correction using adaptive iteratively reweighted penalized least squares. Analyst, 2010; 135(5): 1138-1146.

[19] Mirone P, Chiorboli P. Infrared and Raman spectra and vibrational assignment of maleic anhydride. Spectrochim. Acta, 1962; 18: 1425-1432. 